\title{
Using SMS Text Messaging to Assess Moderators of Smoking Reduction: Validating a New Tool for Ecological Measurement of Health Behaviors
}

\author{
Elliot T. Berkman \\ University of Oregon \\ Emily B. Falk \\ University of Michigan
}

\author{
Janna Dickenson \\ University of California, Los Angeles \\ Matthew D. Lieberman \\ University of California, Los Angeles
}

\begin{abstract}
Objective: Understanding the psychological processes that contribute to smoking reduction will yield population health benefits. Negative mood may moderate smoking lapse during cessation, but this relationship has been difficult to measure in ongoing daily experience. We used a novel form of ecological momentary assessment to test a self-control model of negative mood and craving leading to smoking lapse. Design: We validated short message service (SMS) text as a user-friendly and low-cost option for ecologically measuring real-time health behaviors. We sent text messages to cigarette smokers attempting to quit eight times daily for the first 21 days of cessation $(N$-obs $=3,811)$. Main outcome measures: Approximately every two hours, we assessed cigarette count, mood, and cravings, and examined between- and within-day patterns and time-lagged relationships among these variables. Exhaled carbon monoxide was assessed pre- and posttreatment. Results: Negative mood and craving predicted smoking two hours later, but craving mediated the mood-smoking relationship. Also, this mediation relationship predicted smoking over the next two, but not four, hours. Conclusion: Results clarify conflicting previous findings on the relation between affect and smoking, validate a new low-cost and user-friendly method for collecting fine-grained health behavior assessments, and emphasize the importance of rapid, real-time measurement of smoking moderators.
\end{abstract}

Keywords: smoking cessation, self-control, ecological momentary assessment, text messaging, craving

One of the key issues in studies on cigarette smoking is measuring smoking behavior. The most common measure of smoking is subjective self-assessment, either through global reports or timeline follow-back (TLFB). Each of these depends critically on participants' abilities to accurately remember and recall their own behavior for the course of a day or several days, and in the case of TLFB, with the help of detailed prompts. Despite their wide use, these methods have been shown to be inaccurate due to a number of different cognitive biases (Hammersley, 1994; Shiffman, 2009). For example, cigarette smokers tend to display a "digit bias," or "heap" their reports of smoking around particular numbers reflecting the number of cigarettes per pack or even fractions thereof (e.g., 10 or 20) even though research finds that no such heaping occurs in actual smoking behavior (Klesges, Debon, \& Ray, 1995). Recall of the situational cues for smoking has also been shown to be inaccurate (Shiffman, 1993). Further, some of these biases may

Accepted under the editorial term of Robert M. Kaplan.

Elliot T. Berkman, Department of Psychology, University of Oregon; Janna Dickenson, Department of Psychology, University of California, Los Angeles; Emily B. Falk, Institute for Social Research/Research Center for Group Dynamics, Departments of Communication Studies and Psychology, University of Michigan; Matthew D. Lieberman, Department of Psychology, University of California, Los Angeles.

Correspondence concerning this article should be addressed to Elliot T. Berkman, Department of Psychology, University of Oregon, 1227 University of Oregon, Eugene, OR 97403-1227. E-mail: berkman@uoregon.edu be particularly strong in smokers who are attempting to quit because the emotional antecedents and consequences of relapse can potentiate recall bias (Shiffman et al., 1997). These limitations of recall have led to the consensus that other methods are necessary for accurately assessing smoking and other health-relevant behaviors across time (Collins \& Graham, 2002; Pierce, 2009).

An alternative to retrospective measures is a form of real-time, real-world reporting of behavior called ecological momentary assessment (EMA). The key features of the EMA class of methods are (1) data collection in an ecologically valid environment, (2) real-time or brief interval assessment, and (3) repeated measurements across time and contexts (Shiffman, Stone, \& Hufford, 2008). For example, cigarettes per day can be calculated without recall using EMA by instructing smokers to push a button on a portable pocket-sized device each time they smoke. Cigarette counts derived using this method track biological measures of smoking more reliably than recall methods and do not display digit bias (Shiffman, 2009).

EMA offers a number of additional advantages for studying smokers, smoking, and smoking cessation. Smokers may not always be aware of the extent to which factors such as stress, mood, or urges to smoke impact their smoking (Todd, 2004), and may rely upon heuristics or lay theory if asked to report on the effects directly (Ross, 1989; Ross \& Nisbett, 1991; Tversky \& Kahneman, 1973). In effect, subtle or invisible influences on smoking are less likely to be noticed or deemed relevant. EMA sidesteps this problem by allowing researchers to measure potential moderators 
in real time and calculate their relation to smoking offline. Indeed, studies on smoking antecedents and consequences that have compared real-time to retrospective reports have found only a modest relationship between the two (Delfino, Jamner, \& Whalen, 2001; Shapiro, Jamner, Davyov, \& James, 2002; Shiffman et al., 1997). Furthermore, one study found that within-day, but not betweenday, variations in smoking were predictive of success in a cessation attempt (Chandra, Shiffman, Schafr, Dang, \& Shadel, 2007). These studies converge on the observation that EMA is a valuable tool for identifying important situational moderators of smoking and smoking cessation that might otherwise go unnoticed, and may also translate to understanding the process of engaging in other important health behaviors.

Despite its potential benefits, EMA is not yet widely used to study smoking or health-relevant behaviors because of some practical challenges to its implementation. The primary drawback is that EMA is expensive, typically requiring the purchase of a handheld data collection device (palmtop or personal digital assistant) for each participant (Pierce, 2009; Shiffman et al., 2008). These devices range in cost from around $\$ 75$ to well over $\$ 300$, plus the cost of any software the researcher may want to deploy. There is also an overhead cost in terms of time to install and configure the software for each study. A second limitation is the response burden placed on participants, especially considering that more frequent and closely spaced responses are more statistically reliable than less frequent ones (Collins \& Graham, 2002). A related challenge for participants is the requirement to carry the data collection device during waking hours, often for weeks at a time, and the concomitant risk of missed data when participants forget or choose not to carry the device for a given period. Finally, there is the risk of lost data when devices are lost or destroyed. Though researchers have identified ways to minimize some of these concerns (cf. Graham, Taylor, Olchowski, \& Cumsille, 2006; Shiffman et al., 2008), on balance, the drawbacks are still a major impediment to increased adoption of EMA methods.

A novel way to address several of the remaining disadvantages of EMA is to use short message service (SMS) text messages for data collection instead of customized hardware. Text messages are brief (up to 160 character), low-cost (e.g., $<\$ 0.01$ per message) messages that can be sent to any SMS-enabled cellular telephone. Using text messages would reduce costs substantially relative to other EMA devices because many potential research participants already own SMS-enabled devices. A recent survey found that $86 \%$ of people in the United States have a mobile device and that $91 \%$ of those devices are SMS-enabled, totaling more than 240 million individuals with SMS-enabled phones (Nielsen Mobile, 2010). Participants who do not already own such devices can be provided with prepaid phones which typically cost $\$ 20$ or less. Text messages can be purchased in bulk for less than $\$ 0.01$ each; at this cost, sampling a participant six times each day for a month and receiving responses costs less than $\$ 5$. Moreover, people are increasingly using text messaging, suggesting that potential subjects are already comfortable with the technology. The Nielsen Mobile survey (2010) also revealed that the total traffic volume of text messages has increased by at least $37 \%$ every six months since 2003, and the median text message user sends 200 messages per month. These devices would be ideal for EMA data collection given their low cost and the fact that most people already own and are comfortable using them.
Using text messaging for EMA also may reduce participant burden and data loss. Carrying a cell phone and sending multiple text messages each day has become commonplace in the U.S., so responding to experimental prompts in this way is likely to require minimal subject training and burden. Also, personal cell phones that are used for other reasons in addition to EMA assessment are less likely to be lost or ignored than traditional devices. And critically, many traditional devices store the data collected during the study to be transferred to the experimenter at an endpoint session; devices that are lost or damaged during the study result in data loss for the entire EMA period. Text messaging EMA addresses this problem because data are transmitted to the experimenter as they are entered. This also allows for online quality checking of data as it is being collected.

The purpose of the present study is to validate, for the first time, the use of cell phones for EMA of smoking and smoking antecedents. We do so in the context of the debate regarding the temporal relations between mood and cravings with cigarette smoking, and particularly in the early stages of a cessation attempt when relapse is highly likely (al'Absi, Hatsukami, Davis, \& Wittmers, 2004). Studies have investigated the relation between mood, craving, and smoking during ad libitum smoking and during abstinence, but the relations between those variables is unknown during the early stages of a quitting attempt when smoking may be only reduced before individuals during a quit attempt are able to abstain entirely. For example, some studies of ad libitum smokers suggest that negative mood is prospectively predictive of smoking (Shapiro et al., 2002; Todd, 2004), whereas others find no prospective relationship (Shiffman et al., 2002; Shiffman, Paty, Gwaltney, \& Dang, 2004). Similarly, cravings have been shown to precipitate smoking in the following minutes to hours among non-treatmentseeking smokers (Carter et al., 2008), but not across longer time spans among ex-smokers (Borland \& Blamford, 2005). In terms of cessation, one study found that increased cravings were protective of relapse in the first two weeks of a quitting attempt, but predictive beyond the first two weeks (Herd, Borland, \& Hyland, 2009). Based on these findings, one goal of the present study is to understand the temporal dynamics of how mood and cravings relate to smoking in the early stages of a cessation attempt. In this phase, smokers may not be entirely abstinent and might demonstrate a hybrid of characteristics of ad libitum smokers and of ex-smokers.

It has been suggested that mood and cravings become linked over time as smokers learn that smoking alleviates negative affect, but it is unclear how this linkage may change in the early weeks of a cessation attempt when smokers likely still associate mood and cravings but also are aware of their goal not to smoke (Kassel, Stroud, \& Paronis, 2003; Shiffman,et al., 2007). Identifying the boundary conditions between when and for whom mood and cravings relate to smoking and to each other is particularly relevant for smokers attempting cessation (Shiffman et al., 2007; Shiffman $\&$ Waters, 2004). To do this, we collected data from cigarette smokers who were attempting to quit, eight times each day for the first three weeks following their target quit date. At each time point, approximately every two waking hours, we assessed smoking lapses, cravings, and mood. The relatively fine-grained sampling rate allowed us to examine the effects of mood and cravings on smoking behavior both concurrently and prospectively at a number of prior time points. 


\section{Method}

\section{Participants}

Thirty-one participants ( 15 female) were recruited from smoking cessation programs in Los Angeles via flyers and in-person announcements at the orientation session. All participants were heavy smokers ( $>10$ cigarettes per day, 7 days per week, for at least one year, and urinary cotinine levels of at least $1000 \mathrm{ng} / \mathrm{mL}$ ), and were recruited from professionally led cessation programs (American Lung Association's Freedom From Smoking program). Aside from recruitment, there was no association between the cessation programs and the study. In addition to enrollment in a cessation program, quitting intentions were assessed via scores $>9$ out of 10 on the Contemplation Ladder, a single-item measure of intentions to quit (Biener \& Abrams, 1991), and a cumulative score of at least $18 / 20$ on the Action subscale of the Readiness to Change Questionnaire (RTQ; Rollnick, Heather, Gold, \& Hall, 1992), a 4-item measure of the "action" stage of change. Participants were excluded if they did not speak English, consumed more than 10 alcoholic drinks per week, or had any of the following conditions: dependence on substances other than nicotine, dependence on substances within one year of the baseline session, neurological or psychiatric disorders, cardiovascular disease, or pregnancy. Participants varied in age from 28 to $69(M=46$, $S D=9.7)$, and had been smoking from 11 to 53 years $(M=28.4$, $S D=2.0)$. Participants were ethnically diverse: $52 \%$ were Caucasian, 26\% Hispanic, 19\% African American, and 3\% other. They were compensated $\$ 1$ for each response returned for a possible total of $\$ 168$. Of the original 31 participants, one withdrew from participation and three returned fewer than $50 \%$ of the messages, yielding a total of 27 participants in the analyses reported below. All participants provided written informed consent that was approved by the University of California, Los Angeles Institutional Review Board (IRB).

\section{Procedure}

Phone screening. Following recruitment, participants were contacted via phone to assess intentions to quit with the Contemplation Ladder and RTQ, exclusion criteria, and targeted quit date (TQD). For qualifying participants, a baseline laboratory session was scheduled at least one day prior to the TQD.

Baseline session. Participants came into the lab for a baseline session at least one day prior to their quit date. Following written informed consent, smoking status was confirmed with urinary cotinine (Accutest NicAlert strips; JANT Pharmacal Corp., Encino, CA, U.S.A.), and baseline exhaled carbon monoxide (CO) was measured (Microsmokerlyzer; Bedfont Scientific Ltd., Kent, U.K.). Participants were also screened for amphetamines, cocaine, marijuana, opiates, and PCP with a urine test (Syva RapidTest d.a.u. 5; Dade Behring Inc., Cupertino, CA, U.S.A.). Participants completed measures of demographics, smoking history, waking hours, nicotine dependence (Fagerström Test of Nicotine Dependence, FTND; Heatherton, Kozlowski, Frecker, \& Fagerström, 1991), smoking urges (Questionnaire on Smoking Urges, QSU; Tiffany \& Drobes, 1991), reasons for quitting (Reasons for Quitting Questionnaire, RFQ; Curry, Wagner, \& Grothaus, 1990), and several other questionnaires not relevant to the hypotheses tested here. Participants who did not have or preferred not to use their own cell phones $(N=11)$ were provided with and instructed to use a prepaid phone. Finally, participants were instructed in the use of SMS text messages to receive and respond to prompts, and successfully received and completed a practice prompt.

Ecological momentary assessment. Participants received prompts via text message eight times per day for 21 consecutive days beginning the day prior to their quit date. The first text prompt on each day was sent 15 minutes after morning rise, the last prompt was sent 15 minutes before bedtime, and the other six were spaced evenly throughout the day. Rise and bedtimes were adjusted for each participant separately for weekdays and weekends based on baseline reports of wake time and sleep time. The interprompt interval varied across subjects between 1 hour 50 minutes and 2 hours 25 minutes.

At each prompt, participants responded to three questions: "How many cigarettes have you smoked since the previous signal?" (numerical response), "How much are you craving a cigarette right now?" $(0=$ Not at all, $1=$ A little, $2=$ Somewhat, $3=$ A lot, 4 = Extremely), and "Overall, how is your mood right now?" $(0=$ Extremely negative, $1=$ Somewhat negative, $2=$ Neutral, 3 = Somewhat positive, $4=$ Extremely positive). Participants responded to all three questions with a single text message back to the experimenters using an alphanumeric response code. For example, "0s $1 \mathrm{c} 2 \mathrm{~m}$ " would indicate that the subject has smoked 0 cigarettes, was a " 1 " on the craving scale, and a " 2 " on the mood scale. Errors were handled in the following manner. A typo (e.g., replacing " $r$ " with "s") was assumed if the other two letters were correct. For example, the code " $2 \mathrm{r} 1 \mathrm{c} 3 \mathrm{~m}$ " was recoded as " $2 \mathrm{~s} 1 \mathrm{c} 3 \mathrm{~m}$ ". If two or more letters were incorrect, or if there were repeated letters in the code, the response was treated as missing data. Uninterpretable data was relatively uncommon: there were 9 instances of responses with exactly one typo that was replaced, and 11 instances of uninterpretable data that was treated as missing $(\sim 0.3 \%)$.

Participants could silence or disable their phones at their discretion. In the event that they were unable to respond to a prompt before the arrival of the subsequent prompt, they were instructed to respond only to the most recent prompt. In other words, participants had roughly two hours to respond to each prompt. Participants were sent a reminder text message or received a phone call if their response rate dropped below $50 \%$ for a $24-\mathrm{hr}$ period.

The text message prompts were sent and received through an automated web-based service (RedOxygen Pty. Ltd., Brisbane, Queensland, Australia). Records including the timestamp and content of each message that was sent and received were downloaded from the RedOxygen website.

Endpoint session. An endpoint session was scheduled within seven days of the end of the 21-day EMA period. At this time, exhaled $\mathrm{CO}$ was reassessed along with nicotine dependence (FTND), smoking urges (QSU), and withdrawal symptoms (Shiffman-Jarvik Withdrawal Scale, SJWS; Shiffman \& Jarvik, 1976). Participants were compensated $\$ 1$ for each text message response $(M=\$ 141, S D=\$ 38)$.

\section{Data Analysis Strategy}

Multilevel linear modeling was used to address the nested nature of the data (HLM 6; Scientific Software International, Lincoln- 
wood, IL, U.S.A.; Raudenbush, Bryk, Cheong, \& Congdon, 2004). Specifically, a three-level model was constructed with time points (level-1) nested within days (level-2) nested within participants (level-3). This model allowed us to examine changes in smoking across as well as within days. We examined linear and quadratic slopes of smoking across and within days, as well as patterns of mood and cravings predicting smoking prospectively and concurrently within days. The primary dependent measure of smoking was non-normally distributed because it was reported as a count at each time point with an expected mode of zero. Accordingly, we used a Poisson model with a log link function at the first level that assumes the data are Poisson distributed. All parameters are reported in log-expected likelihood units. Time-series data often violates the assumption of sphericity among the dependent measures. To test for this, we used the Hierarchical Multivariate Linear Modeling module of HLM6 to run a nested set of models. The most unrestricted model allowed for all separate variances and covariances within the $8 \times 8$ within-day variance-covariance matrix, and more restrictive variance structures such as identical variances but unique covariances were nested within that model. Deviance change tests suggested that sphericity was met withindays. Nonetheless, we used robust estimates of standard errors (Zeger, Liang, \& Albert, 1988) with the assumption of overdispersion to conservatively guard against violations of normality and sphericity.

\section{Results}

\section{Response Rate}

Participants responded to $84 \%$ of the prompts on average ( $S D=$ $22 \%, 95 \% \mathrm{CI}=76-92 \%$ ), corresponding to more than six out of eight responses per day. These rates are similar to other experiments (e.g., Shiffman et al., 2007, 86\% in a 2-min time window and Shapiro et al., 2002, 65\% in a 5-min window). Of these responses, $80 \%$ were sent within 23 minutes, and $60 \%$ were sent within 5 minutes. One participant was excluded for responding to fewer than $50 \%$ of the signals; all other participants responded to at least 100 of the 168 prompts (59\%). Within participants, a day was excluded for analysis if it contained fewer than four responses. In total, 90 days were excluded, averaging 3.33 per participant. There were a total of 3,811 level-1 observations, 477 level-2 observations, and 27 level-3 observations.

\section{Correlations Among Global Self-report, EMA Self-report, and CO}

The correlations among the three measures of smoking (logtransformed global self-report, log-transformed ecological momentary assessment self-report, and exhaled carbon monoxide) are shown in Table 1. The correlations between global self-reports of smoking and exhaled CO were .38 and .39 at baseline and endpoint, respectively (both $p \mathrm{~s}<.05$ ). The correlation between calculated total smoking on the last day of EMA (log-transformed) and endpoint $\mathrm{CO}$ was $.50(p<.01$; Figure 1). Endpoint global self-reported smoking and EMA-calculated smoking for the last day were correlated $.90(p<.01)$, and the means differed by 0.98 cigarettes, with global higher than EMA. Consistent with a change
Table 1

Correlations Among Global Self-reports, EMA, and Exhaled CO Measures of Smoking

\begin{tabular}{lcccr}
\hline \multicolumn{1}{c}{ Measure } & 1 & 2 & 3 & 4 \\
\hline $\begin{array}{l}\text { Global smoking self-report of \# } \\
\quad \text { cigarettes smoked (ln) }\end{array}$ & & & & \\
1. Baseline & - & & & \\
$\quad$ 2. Endpoint & .01 & - & & \\
EMA calculation of \# cigarettes & & & & \\
$\quad$ smoked (ln) & .02 & $.90^{* * *}$ & - & \\
$\quad$ 3. Last day \\
Exhaled CO ppm \\
$\quad$ 4. Baseline \\
$\quad$ 5. Endpoint & $.38^{*}$ & -.13 & & -13 \\
\hline
\end{tabular}

Note. $\quad N=27$. Global and EMA self-reports of smoking have been normalized with a natural $\log (\ln )$ transformation. $\mathrm{CO}=$ carbon monoxide; $\mathrm{EMA}=$ ecological momentary assessment.

${ }^{*} p<.05 . \quad{ }^{* * *} p<.01$.

in smoking, there were no significant correlations between any of the baseline and any of the endpoint measures.

\section{Smoking Change From Baseline to Endpoint}

Participants reported smoking 20.2 cigarettes per day $(S D=$ 9.4) at the baseline and 5.2 cigarettes per day $(S D=5.4)$ at the endpoint (change $M=15.0, S E=1.97, t(26)=7.62, p<.01$ ). Nicotine dependence and urges also decreased significantly (see Table 2). Expired CO was marginally reduced, $t(26)=1.94, p=$ .06 (see Table 2), suggesting a reduction in smoking but not complete abstinence. Twelve others abstained for at least one day, nine abstained for two or more days, and eight abstained for three or more days. Only three participants reported remaining abstinent from cigarettes for the duration of the study, and the results reported below remain unchanged if those subjects are excluded from the analyses. In other words, this sample may be characterized as smokers who have reduced their intake as part of a cessation attempt but who are not entirely abstinent. The relatively high rate of lapse is common for smokers in the early weeks of a quitting attempt (Shiffman et al., 2007). As such, this sample represents an ideal opportunity to examine the temporal relations among craving, mood, and smoking in the hybrid stage between ad libitum smoking and cessation (i.e., complete abstinence).

\section{Smoking Patterns Across Days}

The trends in smoking and cravings across days are shown in Figure 2. All results are reported in log-expectation units because a Poisson-link function was used to adjust for he non-normal zero-mode nature of the data. Smoking decreased linearly over time (log-expectation $\gamma=-.04, S E=.011, t(474)=3.45, p<$ .01 ), translating to a $4 \%$ reduction in smoking each day. Similarly, cravings also decreased linearly over time $(\gamma=-.02, S E=.008$, $t(474)=2.40, p<.02)$, translating to a reduction of .02 in cravings (rated on a 5-point scale) each day and more than a half point in the first month. There was no quadratic trend in smoking or cravings when the linear trend was included in the models. In contrast, there was neither a linear nor quadratic trend in mood between days ( $p s n s)$. 


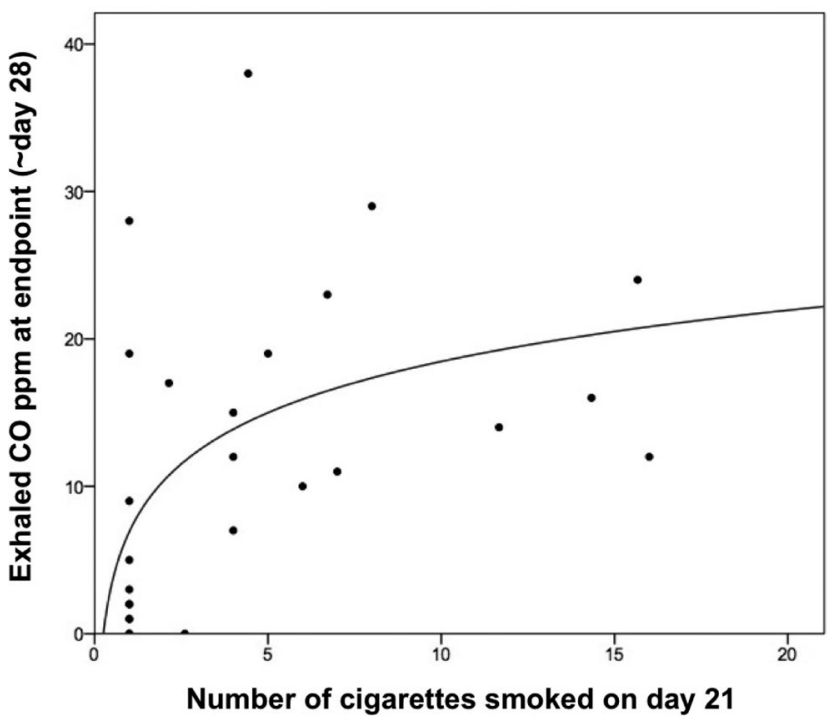

Figure 1. The natural-log relationship between EMA-calculated smoking on Day 21 and exhaled $\mathrm{CO}$ at the endpoint assessment approximately one week later. The logarithmic relationship is significant $\left(F_{1,24}=8.00, p<\right.$ .01 ), and corresponds to a significant linear correlation between logtransformed EMA-calculated smoking and $\mathrm{CO}(r=.50, p<.01)$.

Next, we examined the day-to-day prospective and concurrent relationship between mood and cravings with smoking, controlling for the linear effect across days and nicotine dependence at baseline. Based on the studies reviewed above, we did not expect time-lagged relationships from day to day between mood and smoking. Consistent with this, we found no association between average daily mood and average daily smoking, nor a prospective relationship between average smoking on one day and average mood the next or vice versa. Also consistent with previous studies regarding the early weeks of cessation, average cravings each day related to less average smoking on the subsequent day (logexpectation $\gamma=-.21, S E=.084, t(473)=2.50, p<.01)$, translating to a $17 \%$ reduction in average smoking rate on days following those with cravings 1 point above the mean, for individuals at the mean of nicotine dependence. This effect remained

Table 2

Change From Baseline to Endpoint on Smoking-Related Measures: Mean (SD)

\begin{tabular}{cccr}
\hline Measure & Baseline & Endpoint & Change \\
\hline Global smoking self-report & 20.24 & 5.17 & $15.07^{*}$ \\
(\# cigarettes smoked) & $(9.36)$ & $(5.45)$ & $(10.28)$ \\
Exhaled CO & 18.93 & 13.44 & $5.49^{+}$ \\
(ppm CO) & $(11.65)$ & $(10.89)$ & $(14.70)$ \\
FTND & 6.37 & 2.63 & $3.74^{*}$ \\
(out of 10) & $(2.04)$ & $(2.62)$ & $(2.49)$ \\
Smoking urges (positive) & 4.82 & 2.54 & $2.28^{*}$ \\
(1-7 scale) & $(1.18)$ & $(1.57)$ & $(1.50)$ \\
Smoking urges (negative) & 3.24 & 2.00 & $1.24^{*}$ \\
(1-7 scale) & $(1.21)$ & $(0.97)$ & $(1.35)$ \\
\hline
\end{tabular}

Note. $\quad N=27$

${ }^{+} p=.06 . *{ }^{*} p<.01$.

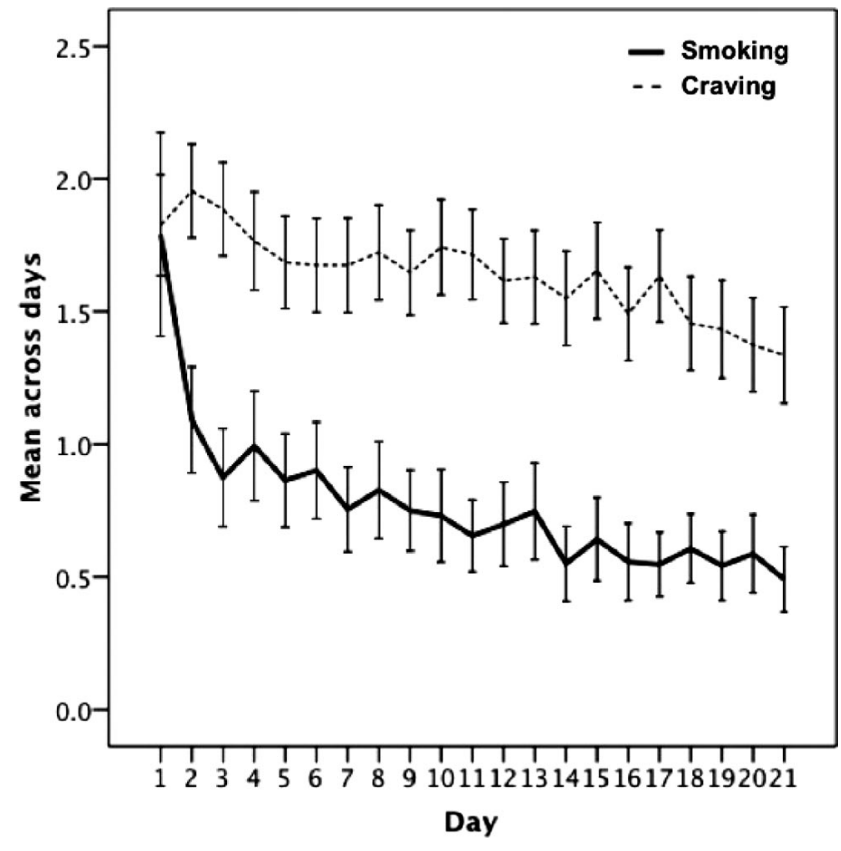

Figure 2. Plot of mean smoking (in number of cigarettes) and mean cravings (from 0-4) at each time point across days. Error bars represent $95 \%$ confidence interval.

significant when controlling for average smoking on the concurrent day, thus cannot be explained by concurrent cravings causing increased smoking on one day and consequently a satiety effect on the following day. In fact, when added to the model, average smoking on one day related to increased smoking on the next day $(\log$-expectation $\gamma=.27, S E=.12, t(472)=2.26, p<.05)$, and the craving to subsequent smoking relationship remained significantly negative.

\section{Smoking Patterns Within Days}

The final set of analyses concerned the patterns of smoking from prompt to prompt within each day. Each of the analyses reported below controls for the linear decline in smoking across days as well as baseline nicotine dependence across individuals to isolate within-person variability in smoking lapses (Gwaltney, Shiffman, Balabnis, \& Paty, 2005).

There were small but significant trends in smoking and mood within days. Participants smoked less in the morning than in the afternoon and evening, resulting in a negative quadratic (but not linear) pattern (log-expectation $\gamma=-.02, S E=.0088, t(3077)=$ 2.26, $p<.03)$. Also, there was a significant and positive linear trend in mood across time $(\gamma=.018, S E=.0075, t(3086)=2.39$, $p<.02$ ), relating to an increase of 0.02 points in mood (on the 5 -point scale) approximately every 2 hours, or .14 points between morning rise and bedtime, and no significant quadratic trend. There was no significant within-day linear or quadratic trend in craving, possibly relating to a mixture of time-of-day smoking profiles in the diverse community sample (Chandra et al., 2007) or to the fact that smokers in the sample appear to be transitioning from heavy smokers to lighter smokers, and these groups have been shown to have distinct daily patterns of smoking (Shiffman \& 
Paty, 2006). In light of the significant trend in smoking across time points within days, all subsequent analyses control for the quadratic effect of time on smoking.

To examine the effect of mood on smoking across time, smoking at time $i$ was simultaneously predicted by concurrent mood (time $i$ ) and previous mood (time $i-1$ ), controlling for the average quadratic within-day trend of smoking (at level 1), the linear trend across days (at level 2), and nicotine dependence (at level 3). In this model, prior negative mood related to increased smoking (log-expectation $\gamma=-.05, S E=.018, t(476)=2.84$, $p<.01$ ), equivalent to a $4.5 \%$ increase in smoking for each point decrease on the 5-point mood scale (Figure 3a). Neither concurrent mood nor mood from more than one time point prior (i.e., time $i-2, i-3$, or $i-4$ ) was related to smoking. ${ }^{1}$

A second model was constructed to examine the relation between concurrent and previous cravings on smoking. As with the model for mood, this model included nicotine dependence and the quadratic within-day and linear between-day trends as covariates. Consistent with the idea that cravings prompts smoking and that smoking satisfies immediate cravings, when both concurrent and prior cravings were entered simultaneously, prior cravings were positively related to concurrent smoking (log-expectation $\gamma=.20$, $S E=.022, t(476)=8.78, p<.01$; Figure $3 \mathrm{~b}$ ), which was negatively related to concurrent cravings (log-expectation $\gamma=$ $-.05, S E=.021, t(476)=2.31, p<.03)$. In other words, for each point increase in the 5-point craving scale at time $i$, smoking was expected to increase by $22 \%$ at time $i+1$, and for each cigarette smoked concurrent cravings were reduced by 0.14 points. As with mood, craving from time $i-2$ was not related to smoking at time $i$.

Based on the suggestion that smokers associate cravings with negative moods because of reinforcement (Kassel et al., 2003), and given that both craving and negative mood at time $i-1$ were related to smoking at time $i$, we ran a final model predicting smoking prospectively from both prior mood and prior craving, controlling for concurrent craving and mood. In this model, prior craving remained significant (log-expectation $\gamma=.23, S E=.024$, $t(476)=9.47, p<.01)$, and the effect of prior mood was not different from zero (log-expectation $\gamma=-.002, S E=.028, p n s)$. Together with a significant negative relationship between concurrent mood and craving $(\gamma=-.30, S E=.073, t(476)=4.10, p<$ $.01)$, this model suggests the possibility that prior craving statistically mediates the relationship between prior mood and smoking. A significant Sobel test of mediation confirmed that prior craving fully mediated the effect of prior mood on smoking $(z=3.79, p<$ .01 ; Figure 4).

\section{Discussion}

This is the first study to capitalize on the massive popularity of text messaging to obtain real-world, real-time, longitudinal assessments of an important health behavior-smoking cessation. We prompted participants to report on their smoking, mood, and craving eight times each day for a period of 21 days. Most of our participants received these prompts on their own SMS-enabled cellular telephones, most of which were already equipped with "unlimited" monthly text messaging plans, minimizing the cost for both hardware and messaging. At $84 \%$, response rates were comparable to those obtained using PDAs employing more elaborate and denser sampling schemes (Shapiro et al., 2002), and attrition was low (13\%), suggesting that participants had little difficulty in receiving and responding to prompts using text messaging.

As expected, we found the relationship of craving and mood to smoking to be dependent on the time scale of the measurement. For example, higher cravings on one day related to less smoking on the next day, but higher cravings at one time point within a day related to more smoking at the next time point. Also, mood and craving predicted smoking roughly two, but not four, hours later. This pattern is in line with prior work showing that affect impacts smoking lapse on a fine-grained time scale within days but not between days (Shiffman \& Waters, 2004). The emerging picture that smoking lapse is highly sensitive to fluctuating intrapersonal and contextual influences underscores the utility of EMA for studying smoking cessation.

We examined the within-day relationship among smoking, craving, and mood to capture some of the dynamic hour-by-hour processes that occur during a quit attempt. Negative mood was not associated with concurrent smoking, but was instead predictive of smoking at the subsequent time point. This pattern is consistent with the idea that during cessation, smoking does not immediately reduce negative mood but smokers continue to smoke in response to negative mood because they believe that it does (Kassel et al., 2003). In further support of this view, negative mood showed a strong concurrent relationship with craving. However, though smoking does not relate to mood, we found that smoking was related to diminished cravings, and, perhaps as a result, cravings were associated with subsequent smoking within days.

Many of the intraday results are encompassed within a mediation model whereby craving mediates the prospective relationship between mood and smoking. Increases in negative mood were concurrently associated with increases in craving, which was in turn associated with subsequent smoking. Identifying this pattern helps to clarify the often inconsistent and perplexing relationship between mood and smoking (Kassel et al., 2003; Shiffman et al., 2007), and suggests that craving may be a better point of intervention for cessation than mood.

It may seem counterintuitive that daily average craving relates negatively to smoking on the next day. In other words, on average across all participants and across all pairs of days, higher cravings on one day relate to reduced smoking on the next, and lower cravings on one day relate to increased smoking the next. This effect was previously found to be unique to the first few weeks of a cessation attempt (Herd et al., 2009), suggesting that smokers are initially able to resist cravings but may fatigue over time (Shiffman \& Waters, 2004). Another important factor in breaking the craving-smoking link is self-efficacy, which has been shown to improve with successful relapse prevention early in a cessation attempt, and is linked to regulating cravings (Borland \& Blamford, 2005). Successfully resisting cravings on one day may lead to increased self-efficacy and thus less smoking the following day. Though this was the pattern for the duration of the present study, it is possible that this pattern would have reversed if we had

\footnotetext{
${ }^{1}$ To examine the relationship between smoking and mood at time $i$, an identical model was run excluding mood at time $i-1$. Smoking related positively, though marginally $(p=.10)$ to concurrently reported negative $\operatorname{mood}(\gamma=-.03, S E=.017, t(476)=1.63, p<.10)$.
} 


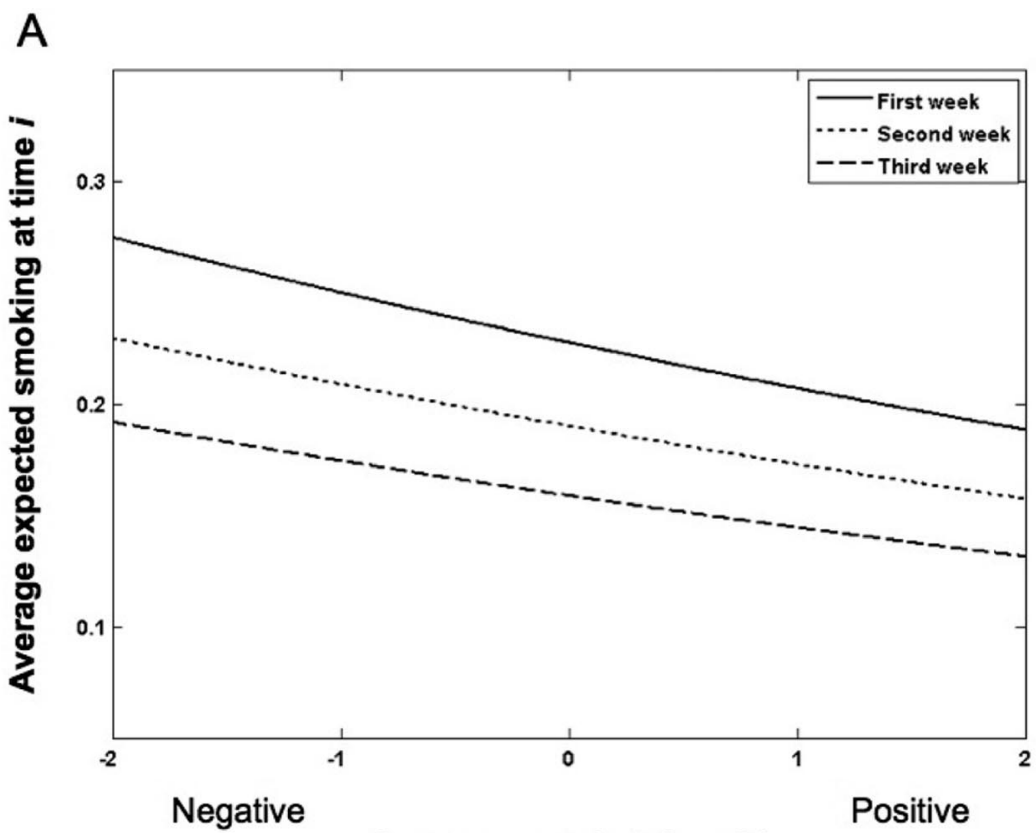

B

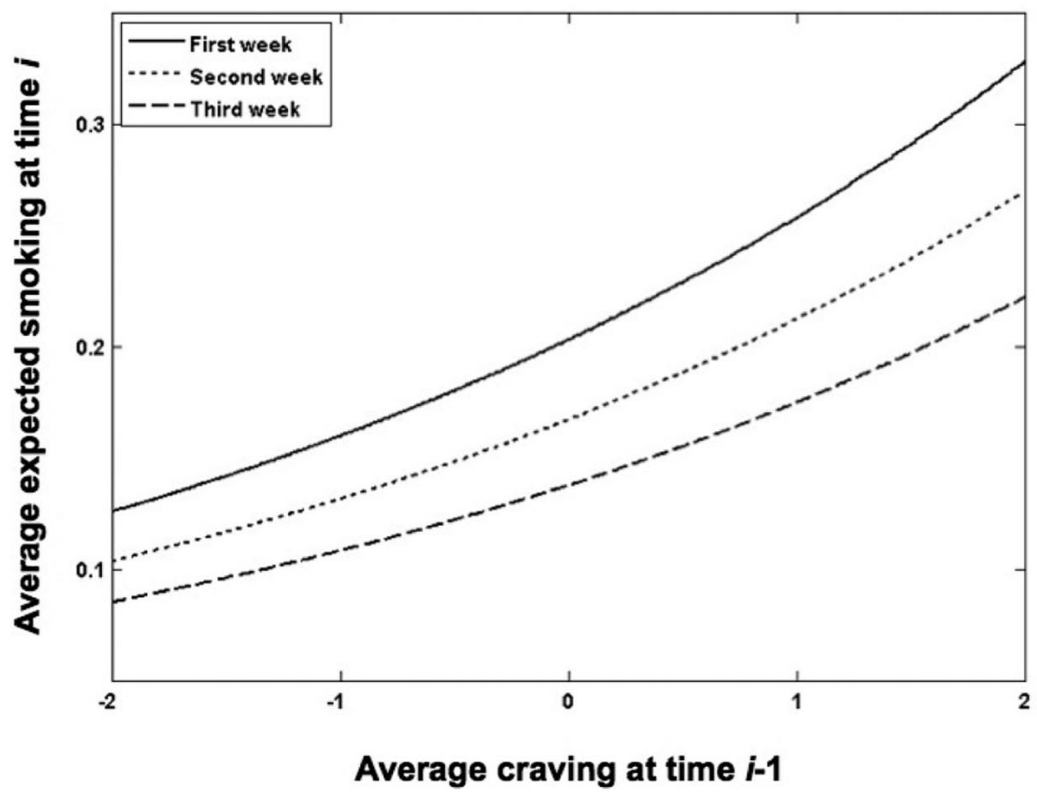

Figure 3. The relationship between prior mood (A) and prior cravings (B) on concurrent smoking, controlling for the quadratic trend within days, the linear effect between days, and baseline nicotine dependence. (A) Mood at time $i-1$ predicting smoking at time $i$, controlling for time $i$ mood, is a significant predictor of smoking $(\log$-expectation $\gamma=-.05, t(476)=2.84, p<.01)$. A one-point decrease on the 5-point mood scale (i.e., more negative mood) related to $4.5 \%$ increase in smoking at the following time point. (B) Craving at time $i-1$ predicting smoking at time $i$, controlling for time $i$ craving, is a significant predictor of smoking (log-expectation $\gamma=.20, t(476)=8.78, p<.01$ ). A one-point increase on the 5-point craving scale (i.e., higher cravings) related to $22 \%$ increase in smoking at the following time point.

observed our sample at a different period of time (e.g., 4-8 weeks following cessation). Also, it is interesting to note that the relationship between craving and smoking is different between days than it is within days. In addition to suggesting that there are different mechanisms behind the craving-smoking relationship that vary by time scale, this finding further underscores the importance of examining both within- and between-day variability in outcomes during smoking cessation. 


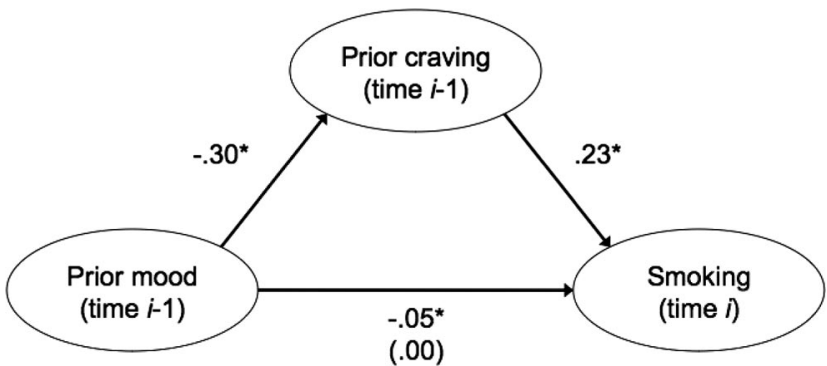

Figure 4. Craving mediates the within-day relationship between negative mood and smoking. Negative mood relates to increased smoking at the following time point (Figure 4a), as does craving (Figure 4b). Negative mood is also associated with craving concurrently $(\gamma=-.30, t(476)=$ $4.10, p<.01)$. When entered simultaneously, prior craving significantly relates to smoking (log-expectation $\gamma=.23, t(476)=9.47, p<.01)$ but prior mood does not $(p n s)$, suggesting full mediation (Sobel's $z=3.79$, $p<.01)$.

One limitation of the present study is the lack of comparison groups. It would have improved the study if there were a group of smokers that received text messages but not about smoking, and another group that received no text messages. These groups would have helped isolate the effects of repeatedly considering one's own experience during a cessation attempt and of repeatedly sending text messages throughout the day, respectively. However, there are reasons to believe that these effects would be minimal. Several other EMA studies have found no reactivity effects between groups of do and do not receive prompts throughout the day (Hufford \& Shields, 2002; Hufford, Shields, Shiffman, Paty, \& Balabanis, 2002; Stone et al., 2003), and if anything, text messaging is likely to be less disruptive than assessments using an unfamiliar device. In fact, it has become normative to send and receive at least eight text messages among people who have cell phones (Nielsen Mobile, 2010), suggesting that our participants were already accustomed to attending to text messages repeatedly throughout the day. Further, the use of HLM controls for day- and person-level effects to isolate within-day sources of variance. It seems unlikely that any methodological effects would change so rapidly within days. Regardless, the reactance effects of this new technology remain unknown and an important target for future studies.

It is important to note that the findings reported here might be limited to a particular phase in smoking cessation: one in which smokers are actively attempting to quit but are not abstinent from smoking. This phase is distinct from ad libitum smoking because participants have reduced intake, and also distinct from smoking lapse because participants continue (reduced) smoking. For many smokers, this phase seems to serve as an intermediate or transition phase between ad libitum smoking and complete abstinence (e.g., see high relapse rates before abstinence in Shiffman et al., 2007). This phase may be exactly when participants begin to alter the habitual associations between mood/craving and smoking as they to transition from ad libitum to ex-smokers. This would partially explain the inconsistent results among studies examining the early phases of quitting. If this is the case, this early transition phase might prove to be an important area for further study. For example, it seems likely that some patterns in the craving-mood-smoking relationship could be more or less predictive of subsequent prolonged abstinence.

To the extent that text messaging can be used in studies on smoking and smoking cessation, it is important to note some of its limitations. First, the present paradigm required participants to recall smoking across the past two hours and sometimes more if a signal was missed. Future studies should capitalize even further on the benefits of text messaging to reduce memory biases by increasing the frequency of assessments. And second, user input is somewhat limited compared to other electronic methods (e.g., email or web), which restricts the complexity of survey items. These weaknesses only highlight the areas where text messaging is strongest: high-frequency responses to simple questions. As such, smoking is an ideal candidate for text messaging-based studies.

Future studies can also build on the current study by developing cessation interventions that use text messaging. As this study and others have documented, the proximal predictors of relapse are not necessarily major life events but instead small within-day fluctuations in the social environment and the intrapsychic milieu (e.g., Borland \& Blamford, 2005; Shiffman et al., 2007). Furthermore, there is growing consensus on the efficacy of tailored interventions for smoking cessation (Chua, Liberzon, Welsh, \& Strecher, 2009; Dijkstra, 2005; Strecher, 1999; Strecher, Shiffman, \& West, 2006), and two studies have already successfully used text messaging for smoking cessation intervention, showing a twofold increase in self-reported quit rates across six weeks (Free et al., 2009; Rodgers et al., 2005). Text messaging may be an ideal delivery mechanism for tailored interventions because it is low-cost, most people already possess the existing hardware, and the messages can be delivered near-instantaneously into real-world situations. For example, participants' daily fluctuations in mood and craving could be measured for a week before the cessation attempt; then during cessation, tailored messages could be sent automatically at times each day when cravings were known to be high. We believe that the current study demonstrates the utility of text messaging for smoking cessation and other health research and interventions more broadly, and anticipate that future work will capitalize on the unique potential of this growing technology.

\section{References}

al'Absi, M., Hatsukami, D., Davis, G.L., \& Wittmers, L.E. (2004). Prospective examination of effects of smoking abstinence on cortisol and withdrawal symptoms as predictors of early smoking relapse. Drug and Alcohol Dependence, 73(3), 267-278.

Biener, L., \& Abrams, D. B. (1991). The Contemplation Ladder: Validation of a measure of readiness to consider smoking cessation. Health Psychology, 10, 360-365.

Borland, R., \& Balmford, J. (2005). Perspectives on relapse prevention: An exploratory study. Psychology and Health, 20, 661-671.

Carter, B., Lam, C., Robinson, J., Paris, M., Waters, A., Wetter, D., \& Cinciripini, P.M. (2008). Real-time craving and mood assessments before and after smoking. Nicotine \& Tobacco Research, 10, 1165-1169.

Chandra, S., Shiffman, S., Scharf, D. M., Dang, Q., \& Shadel, W. G. (2007). Daily smoking patterns, their determinants, and implications for quitting. Experimental and Clinical Psychopharmacology, 15, 67-80.

Chua, H., Liberzon, I., Welsh, R., \& Strecher, V. (2009). Neural correlates of message tailoring and self-relatedness in smoking cessation programming. Biological Psychiatry, 65, (165-168).

Collins, L. M., \& Graham, J. W. (2002). The effect of the timing and spacing of observations in longitudinal studies of tobacco and other drug 
use: Temporal design considerations. Drug \& Alcohol Dependence, 68, S85-S96.

Curry, S., Wagner, E. H., \& Grothaus, L. C. (1990). Intrinsic and extrinsic motivation for smoking cessation. Journal of Consulting and Clinical Psychology, 58, 310-316.

Delfino, R. J., Jamner, L. D., \& Whalen, C. K. (2001). Temporal analysis of the relationship of smoking behavior and urges to mood states in men versus women. Nicotine \& Tobacco Research, 3, 235-248.

Dijkstra, A. (2005). Working mechanisms of computer-tailored health education: Evidence from smoking cessation. Health Education Research, 20, 527-539.

Free, C., Whittaker, R., Knight, R., Abramsky, T., Rodgers, A., \& Roberts, I. G. (2009). Txt2stop: A pilot randomised controlled trial of mobile phone-based smoking cessation support. Tobacco Control, 18, 88-91.

Graham, J. W., Taylor, B. J., Olchowski, A. E., \& Cumsille, P. E. (2006). Planned missing data designs in psychological research. Psychological Methods, 11, 323-343.

Gwaltney, C. J., Shiffman, S., Balabanis, M. H., \& Paty, J. A. (2005). Dynamic self-efficacy and outcome expectancies: Prediction of smoking lapse and relapse. Journal of Abnormal Psychology, 114, 661-675.

Hammersley, R. (1994). A digest of memory phenomena for addiction research. Addiction, 89, 283-293.

Heatherton, T. F., Kozlowski, L. T., Frecker, R. C., \& Fagerström, K. O. (1991). The Fagerström Test for Nicotine Dependence: A revision of the Fagerström Tolerance Questionnaire. British Journal of Addiction, 86, $1119-1127$.

Herd, N., Borland, R., \& Hyland, A. (2009). Predictors of smoking relapse by duration of abstinence: Findings from the International Tobacco Control (ITC) Four Country Survey. Addiction, 104, 2088-2099.

Hufford, M. R., \& Shields, A. L. (2002). Electronic diaries: An examination of applications and what works in the field. Applied Clinical Trials, $11,46-56$.

Hufford, M. R., Shields, A. L., Shiffman, S., Paty, J., \& Balabanis, M. (2002). Reactivity to ecological momentary assessment: An example using undergraduate problem drinkers. Psychology of Addictive Behaviors, 16, 205-211.

Kassel, J. D., Stroud, L. R., \& Paronis, C. A. (2003). Smoking, stress, and negative affect: Correlation, causation, and context across stages of smoking. Psychological Bulletin, 129. 270-304.

Klesges, R. C., Debon, M., \& Ray, J. W. (1995). Are self-reports of smoking rate biased? Evidence from the second national health and nutrition examination survey. Journal of Clinical Epidemiology, 48, $1225-1233$

Nielsen Mobile. (2010). Nielsen Telecom practice group report on text messaging, 2010. The Nielsen Wire, New York, NY.

Pierce, J. P. (2009). Electronic recording, self-report, and bias in measuring cigarette consumption. Health Psychology, 28, 527-528.

Raudenbush, S. W., Bryk, A. S., Cheong, Y. F., \& Congdon, R. (2004). HLM6: Hierarchical linear and nonlinear modeling. Lincolnwood, IL: Scientific Software International.

Rodgers, A., Corbett, T., Bramley, D., Riddell, T., Wills, M., Lin, R.-B., \& Jones, M. (2005). Do u smoke after txt? Results of a randomised trial of smoking cessation using mobile phone text messaging. Tobacco Control, 14, 255-261.

Rollnick, S., Heather, N., Gold, R., \& Hall, W. (1992). Development of a short 'readiness to change' questionnaire for use in brief, opportunistic interventions among excessive drinkers. British Journal of Addiction, 87, 743-754.
Ross, L., \& Nisbett, R. E. (1991). The person and the situation: Perspectives of social psychology. New York: McGraw-Hill.

Ross, M. (1989). Relation of implicit theories to the construction of personal histories. Psychological Review, 96, 341-357.

Shapiro, D., Jamner, L. D., Davydov, D. M., \& James, P. (2002). Situations and moods associated with smoking in everyday life. Psychology of Addictive Behaviors, 16, 342-345.

Shiffman, S. (1993). Assessing smoking patterns and motives. Journal of Consulting and Clinical Psychology, 61(5), 732-742.

Shiffman, S. (2009). Commentary on Herd \& Borland (2009) and Herd et al. (2009): Illuminating the course and dynamics of smoking cessation. Addiction, 104, 2100-2101.

Shiffman, S., Balabanis, M. H., Gwaltney, C. J., Paty, J. A., Gnys, M., Kassel, J. D., . . Paton, S. M. (2007). Prediction of lapse from associations between smoking and situational antecedents assessed by ecological momentary assessment. Drug \& Alcohol Dependence, 91, 159-168.

Shiffman, S., Gwaltney, C. J., Balabanis, M. H., Liu, K. S., Paty, J. A., Kassel, J. D., ... Gnys, M. (2002). Immediate antecedents of cigarette smoking: An analysis from ecological momentary assessment. Journal of Abnormal Psychology, 111, 531-543.

Shiffman, S., Hufford, M., Hickcox, M., Paty, J. A., Gnys, M., \& Kassel, J. D. (1997). Remember that? A comparison of real-time versus retrospective recall of smoking lapses. Journal of Consulting and Clinical Psychology, 65, 292-300.

Shiffman, S., Paty, J. A., Gwaltney, C. J., \& Dang, Q. (2004). Immediate antecedents of cigarette smoking: An analysis of unrestricted smoking patterns. Journal of Abnormal Psychology, 113, 166-171.

Shiffman, S., Paty, J. A. (2006). Smoking patterns and dependence: Contrasting chippers and heavy smokers. Journal of Abnormal Psychology, 115, 509-523.

Shiffman, S., Stone, A. A., \& Hufford, M. R. (2008). Ecological momentary assessment. Annual Review of Clinical Psychology, 4, 1-32.

Shiffman, S., \& Waters, A. J. (2004). Negative affect and smoking lapses: A prospective analysis. Journal of Consulting and Clinical Psychology, 72, 192-201.

Shiffman, S. M., \& Jarvik, M. E. (1976). Smoking withdrawal symptoms in two weeks of abstinence. Psychopharmacology, 50, 35-39.

Stone, A. A., Broderick, J., Schwartz, J., Shiffman, S., Litcher-Kelly, L., \& Calvanese, P. (2003). Intensive momentary reporting of pain with an electronic diary: Reactivity, compliance, and patient satisfaction. Pain, 104, 343-351.

Strecher, V. J. (1999). Computer-tailored smoking cessation materials: A review and discussion. Patient Education and Counseling, 36, 107-117.

Strecher, V. J., Shiffman, S., \& West, R. (2006). Moderators and mediators of a Web-based computer-tailored smoking cessation program among nicotine patch users. Nicotine \& Tobacco Research, 8, S95-S101.

Tiffany, S. T., \& Drobes, D. J. (1991). The development and initial validation of a questionnaire on smoking urges. British Journal of Addiction, 86, 1467-1476.

Todd, M. (2004). Daily processes in stress and smoking: Effects of negative events, nicotine dependence, and gender. Psychology of Addictive Behaviors, 18, 31-39.

Tversky, A., \& Kahneman, D. (1973). Availability: A heuristic for judging frequency and probability. Cognitive Psychology, 5, 207-232.

Zeger, S. L., Liang, K.-Y., \& Albert, P. S. (1988). Models for longitudinal data: A generalized estimating equation approach. Biometrics, 44, $1049-1060$ 